JOURNAL OF INTEGRAL EQUATIONS

AND APPLICATIONS

Volume 9, Number 3, Summer 1997

\title{
THE SOLUTION IN A CLASS OF SINGULAR FUNCTIONS OF CAUCHY TYPE BISINGULAR INTEGRAL EQUATIONS
}

\author{
D. ELLIOTT, I.K. LIFANOV AND G.S. LITVINCHUK
}

\begin{abstract}
We first give the solution, in a class of singular functions, of a one-dimensional singular integral equation with Cauchy integral, arising from a problem in two-dimensional aerodynamics. The analysis is then extended to the solution of a bisingular Cauchy singular integral equation arising from three-dimensional airfoil theory.
\end{abstract}

1. Introduction. Traditionally, the theory of singular integral equations with Cauchy kernel has been constructed under the assumption that the unknown function is absolutely integrable. See, for example, the texts by Muskhelishvili [7] or Gakhov [4]. Such theory has immediate application in the mathematical theories of elasticity and hydrodynamics. But airfoil control problems lead to the necessity of introducing an ejection external stream emanating from a point or a line on the airfoil which can be modeled by the introduction of a source into the airfoil; see Belotserkovsky and Lifanov [2] and Woolard [9]. In the simplest case, this modeling leads to the necessity of solving a singular integral equation with Cauchy kernel on an interval, with the solution having a non-integrable singularity of the form $1 / x$ near $x=0$. The so-called method of discrete vortices has been applied by Lifanov, Mikhailov and Titsky [6] for the case of incompressible flow past an airfoil, with a slit in the airfoil possessing a source. An explicit analytic solution to this problem is not known but Bisplinghoff, Ashley and Halfman [3] have shown that in the case of a rectangular wing, the problem is reduced to that of solving a bisingular integral equation of the first kind with Cauchy integrals taken over the Cartesian product of two finite intervals. We shall show that, in a special case, it is possible to write down explicitly all the solutions that are absolutely integrable. In addition, we shall also give analytic solutions of this equation having

\footnotetext{
Received by the editors on June 1, 1996, and in revised form on April 8, 1997.

This project has been partially supported by a grant from the Australian Research Council.

Copyright (C)1997 Rocky Mountain Mathematics Consortium
} 
a non-integrable singularity of the form $1 /(x-q)$ at some point $q$ in $(-1,1)$. We might note in passing that such singular solutions in the one-dimensional case have been used by Zakharov, Lifanov and Lifanov [10] for diffraction problems of electromagnetic waves.

Let an airfoil $L$ be described by a plane simple curve $x=x(t)$, $y=y(t), t \in[0, l]$ in the $x y$ plane. We shall assume that $L$ is either an arc or a closed contour with $x^{\prime}(t), y^{\prime}(t)$ satisfying the Hölder condition $H(\alpha), 0<\alpha \leq 1$, on $L$ (see below, (2.3)). Furthermore, it will be assumed that, at any point $M(t)=M(x(t), y(t))$ on $L$, $r_{M}^{\prime}:=\left(x^{\prime 2}(t)+y^{\prime 2}(t)\right)^{1 / 2} \neq 0$ for $0 \leq t \leq l$.

Now let the airfoil be immersed in a steady incompressible flow with free stream velocity $\vec{U}_{0}$. The surface of the airfoil will be modeled by a vortex sheet whose density at the point $M(t)$ of $L$ is given by $\gamma(t)$, see [1] and [2]. It is known that the velocity $\vec{V}\left(M_{0}\right)$ at a point $M_{0}$ arising from the vorticity layer on $L$ is defined by

$$
\begin{aligned}
\vec{V}\left(M_{0}\right) & =\int_{L} \vec{\omega}\left(t_{0}, t\right) \gamma(t) d s \\
& =\frac{1}{2 \pi} \int_{L} \frac{y\left(t_{0}, t\right) \hat{\mathbf{i}}-x\left(t_{0}, t\right) \hat{\mathbf{j}}}{r_{M M_{0}}^{2}} \gamma(t) d s
\end{aligned}
$$

where $s$ denotes the arc length along $L$. Here

$$
\begin{aligned}
x\left(t_{0}, t\right) & :=x\left(t_{0}\right)-x(t), \\
y\left(t_{0}, t\right) & :=y\left(t_{0}\right)-y(t), \\
M_{0} \notin L \quad \text { and } \quad r_{M M_{0}} & :=\left|\mathbf{r}_{M M_{0}}\right|:=\left|x\left(t_{0}, t\right) \hat{\mathbf{i}}+y\left(t_{0}, t\right) \hat{\mathbf{j}}\right| .
\end{aligned}
$$

As $t_{0}$ approaches the curve $L$, then

$$
\vec{V}^{ \pm}\left(M_{0}\right)= \pm(1 / 2) \gamma\left(t_{0}\right) \hat{\boldsymbol{\tau}}\left(t_{0}\right)+\int_{L} \vec{\omega}\left(t_{0}, t\right) \gamma(t) d s
$$

where $\hat{\boldsymbol{\tau}}\left(t_{0}\right)$ is the unit tangent at the point $M_{0}$ and the sign \pm is chosen according to whether we approach $L$ from the direction of the outward or inward normal, respectively. Equation (1.2) shows that the normal component of velocity, $\hat{\mathbf{n}}_{M_{0}} \cdot \vec{V}\left(M_{0}\right)$ where $\hat{\mathbf{n}}_{M_{0}}:=\left(\hat{\mathbf{i}} y^{\prime}\left(t_{0}\right)-\hat{\mathbf{j}} x^{\prime}\left(t_{0}\right)\right) / r_{M_{0}}^{\prime}$ is the outward drawn unit normal at $M_{0}$, is continuous as we pass through the curve $L$. Therefore, the condition of no flow across the 
airfoil, i.e., the sum of the normal components of the free stream and of the vorticity layer at the point $M_{0}$ of $L$, gives

$$
\int_{L} \vec{\omega}\left(t_{0}, t\right) \cdot \hat{\mathbf{n}}_{M_{0}} \gamma(t) d s=-\vec{U}_{0}\left(M_{0}\right) \cdot \hat{\mathbf{n}}_{M_{0}}
$$

$M_{0} \in L$. If we assume that the parameter $t$ coincides with the arc length $s$ of $L$, then (1.3) can be written as

$$
\frac{1}{2 \pi} \int_{L} \frac{x^{\prime}\left(t_{0}\right) x\left(t_{0}, t\right)+y^{\prime}\left(t_{0}\right) y\left(t_{0}, t\right)}{r_{M M_{0}}^{2}} \gamma(t) d t=-\vec{U}_{0}\left(M_{0}\right) \cdot \hat{\mathbf{n}}_{M_{0}}
$$

Equation (1.4) is a singular integral equation of the first kind, with Cauchy kernel when $L$ is an arc and Hilbert kernel if $L$ is a contour.

Suppose now that at a point $M_{Q}\left(x\left(t_{Q}\right), y\left(t_{Q}\right)\right.$ ) of $L$ (which is not an end-point if $L$ is an arc) there is a device of external flow ejection on the upper side of the airfoil which does not perturb the velocities on the lower sides of the airfoil. We shall model the ejection device by a source of intensity $Q$, see [5]. Thus, on the upper side of the airfoil, the velocity field in the neighborhood of $M_{Q}$ will be defined by a source and have a singularity, but on the lower side the field has no singularities. Again, the surface of the airfoil is modeled by a vorticity layer when we have a free stream and a source on the airfoil. The condition of no flow across the airfoil, cf. equation (1.3), now gives

$$
\int_{L} \vec{\omega}\left(t_{0}, t\right) \cdot \hat{\mathbf{n}}_{M_{0}} \gamma(t) d t=-\vec{U}_{0} \cdot \hat{\mathbf{n}}_{M_{0}}-\vec{V}_{Q} \cdot \hat{\mathbf{n}}_{M_{0}}
$$

for $M_{0} \in L, M_{0} \neq M_{Q}$ where

$$
\begin{aligned}
\vec{V}_{Q}(M) & =\frac{Q}{2 \pi} \cdot \frac{\mathbf{r}_{M M_{Q}}}{r_{M M_{Q}}^{2}} \\
\vec{V}_{Q}\left(M_{0}\right) \cdot \hat{\mathbf{n}}_{M_{0}} & =\frac{Q}{2 \pi} \cdot \frac{y^{\prime}\left(t_{0}\right) x\left(t_{Q}, t_{0}\right)-x^{\prime}\left(t_{0}\right) y\left(t_{Q}, t_{0}\right)}{r_{M_{0} M_{Q}}^{2}} .
\end{aligned}
$$

Using the result of [1] , it is possible to show that the function $\vec{V}_{Q} \cdot \hat{\mathbf{n}}_{M_{0}}$ has no singularity at the point $M_{Q}$ and moreover it belongs to the class $H(\alpha)$ on $[0, l]$ if $x^{\prime \prime}(t)$ and $y^{\prime \prime}(t) \in H(\alpha)$. 
Let us now consider the class of functions necessary to solve equation (1.5). Since the free stream and normal component of velocity from the source do not have singularities on both sides of the airfoil, on the lower side of the airfoil the function

$$
\psi(t)=\vec{V}^{-}(t) \cdot \hat{\boldsymbol{\tau}}_{M}(t)+\vec{V}_{Q}(t) \cdot \hat{\boldsymbol{\tau}}_{M}(t)
$$

cannot have a singularity. From (1.6), it follows that the function $\vec{V}_{Q}(t) \cdot \hat{\boldsymbol{\tau}}_{M}$ has, at the point $M_{Q}$, a singularity of the form $1 /\left(t-t_{Q}\right)$. Hence the function $\vec{V}^{-} \cdot \hat{\tau}_{M}$ has the same singularity. It may be shown, see [5] and [7], that in a neighborhood of the point $t_{Q}$ we have

$$
\begin{gathered}
\vec{V}_{Q}(t) \cdot \hat{\tau}_{M}=\frac{Q}{2 \pi} \cdot \frac{1}{t_{Q}-t}+D\left(t_{Q}, t\right) \\
\vec{V}^{-}(t) \cdot \hat{\tau}_{M}(t)=-\frac{\gamma(t)}{2}+\frac{1}{2 \pi} \\
\cdot \int_{L} \frac{x^{\prime}(t) y\left(t, t_{0}\right)-y^{\prime}(t) x\left(t, t_{0}\right)}{r_{M M_{0}}^{2}} \gamma\left(t_{0}\right) d t_{0} .
\end{gathered}
$$

As mentioned above, if $x^{\prime \prime}(t), y^{\prime \prime}(t) \in H(\alpha)$ on $[0, l]$, then the kernel in (1.9) is smooth and belongs to $H(\alpha)$ also. The same is true of the function $D\left(t_{Q}, t\right)$. Therefore, if we choose the function $\gamma(t)$ so that in a neighborhood of the point $t_{Q}$ it has the form

$$
\gamma(t)=\frac{Q}{\pi} \cdot \frac{1}{t_{Q}-t}+\eta(t),
$$

where $\eta$ is smooth, then (1.7) follows on the strength of equations (1.8) and (1.9).

From all of this we conclude that, for the solution of the physical problem with ejection of the external flow at a point $t_{Q}$ on the upper side of the airfoil, we must solve equation (1.5) in the class of functions given by (1.10).

2. One-dimensional singular integral equations of the first kind on a finite interval. Let us first recall the solutions of the airfoil equation

$$
\frac{1}{\pi} \int_{-1}^{1} \frac{\gamma(\xi) d \xi}{\xi-x}=f(x), \quad x \in(-1,1)
$$


where the Cauchy principal value integral is defined by

$$
\int_{-1}^{1} \frac{\gamma(\xi) d \xi}{\xi-x}:=\lim _{\varepsilon \rightarrow 0+}\left(\int_{-1}^{x-\varepsilon}+\int_{x+\varepsilon}^{1}\right) \frac{\gamma(\xi) d \xi}{\xi-x},
$$

provided that the integral exists. Furthermore, we shall assume that $f$ is Hölder continuous of Hölder index $\alpha, 0<\alpha \leq 1$, on $[-1,1]$. That is, for any $x_{1}, x_{2} \in[-1,1]$, we have

$$
\left|f\left(x_{1}\right)-f\left(x_{2}\right)\right| \leq L\left|x_{1}-x_{2}\right|^{\alpha},
$$

where $L$ is a constant independent of $x_{1}$ and $x_{2}$. We write $f \in H(\alpha)$.

We have the following situations:

1. Suppose $\gamma$ is bounded at both end-points \pm 1 . Such solutions are said to be of index -1 and are given by

$$
\gamma(x)=-\frac{\left(1-x^{2}\right)^{1 / 2}}{\pi} \int_{-1}^{1} \frac{f(\xi) d \xi}{\left(1-\xi^{2}\right)^{1 / 2}(\xi-x)},
$$

provided that $f$ is such that

$$
\int_{-1}^{1} \frac{f(\xi) d \xi}{\left(1-\xi^{2}\right)^{1 / 2}}=0
$$

2. Suppose $\gamma$ is bounded at one end and unbounded at the other although absolutely integrable there. Such solutions are said to be of index 0 and are given by either

$$
\gamma(x)=-\frac{1}{\pi}\left(\frac{1-x}{1+x}\right)^{1 / 2} \int_{-1}^{1}\left(\frac{1+\xi}{1-\xi}\right)^{1 / 2} \frac{f(\xi) d \xi}{\xi-x},
$$

or

$$
\gamma(x)=-\frac{1}{\pi}\left(\frac{1+x}{1-x}\right)^{1 / 2} \int_{-1}^{1}\left(\frac{1-\xi}{1+\xi}\right)^{1 / 2} \frac{f(\xi) d \xi}{\xi-x}
$$

depending upon whether $\gamma$ is to be bounded at +1 or -1 , respectively. 
3. Suppose $\gamma$ is unbounded at both end-points but absolutely integrable. Such solutions are said to be of index 1 and are given by

$$
\begin{aligned}
\gamma(x)= & -\frac{1}{\left(1-x^{2}\right)^{1 / 2}} \frac{1}{\pi} \int_{-1}^{1} \frac{\left(1-\xi^{2}\right)^{1 / 2} f(\xi) d \xi}{\xi-x} \\
& +\frac{A}{\pi\left(1-x^{2}\right)^{1 / 2}},
\end{aligned}
$$

where $A$ is an arbitrary constant. The value of $A$ may be determined by imposing a further condition such as, for example,

$$
\int_{-1}^{1} \gamma(\xi) d \xi=A_{1}
$$

Remark 2.1. Suppose that the righthand side of (2.1) depends on a further param eter so that it is given by $f(x, y)$ where $-1 \leq y \leq 1$. If $f(x, y)$ is Hölder continuous, of index $\alpha$, on $I_{c}^{2}:=[-1,1] \times[-1,1]$, then all the previous results remain valid provided that the constant $A$ in (2.8) is replaced by $A(y)$. Note that $f(x, y)$ is Hölder continuous of Hölder index $\alpha, 0<\alpha \leq 1$, on $I_{c}^{2}$ if

$$
\left|f\left(x_{1}, y_{1}\right)-f\left(x_{2}, y_{2}\right)\right| \leq L\left\{\left|x_{1}-x_{2}\right|^{\alpha}+\left|y_{1}-y_{2}\right|^{\alpha}\right\}
$$

for some $L$ independent of $x_{1}, y_{1}, x_{2}$ and $y_{2}$.

Let $q$ be a given point of $(-1,1)$. We shall now look for all solutions of equation (2.1) which are of the form

$$
\gamma(x)=\phi(x)+\psi(x) /(q-x),
$$

for $x \in(-1, q) \cup(q, 1)$, where $\phi$ and $\psi$ are in the class $H^{*}$ of Muskhelishvili [7, p. 238]. Such functions are Hölder continuous on every closed interval of $(-1,1)$ and absolutely integrable at the endpoints \pm 1 (see also Okada and Elliott [8]). In the remainder of this paper, we shall denote by $H_{q}^{*}$ the class of functions of the form given by equation (2.9).

Theorem 2.1. Suppose $f \in H(\alpha), 0<\alpha \leq 1$, on $[-1,1]$. Solutions of (2.1) in the class $H_{q}^{*}(\alpha),-1<q<1,0<\alpha \leq 1$, are given as follows. 
1. If $\gamma$ is bounded at both endpoints \pm 1 ,

$$
\begin{aligned}
\gamma(x)= & -\frac{\left(1-x^{2}\right)^{1 / 2}}{\pi} \int_{-1}^{1} \frac{f(\xi) d \xi}{\left(1-\xi^{2}\right)^{1 / 2}(\xi-x)} \\
& +\frac{\left(1-x^{2}\right)^{1 / 2}}{q-x} \cdot \frac{1}{\pi} \int_{-1}^{1} \frac{f(\xi) d \xi}{\left(1-\xi^{2}\right)^{1 / 2}} .
\end{aligned}
$$

2. If $\gamma$ is bounded at one endpoint and unbounded at the other, although absolutely integrable there, then either

$$
\begin{aligned}
\gamma(x)= & -\frac{1}{\pi}\left(\frac{1-x}{1+x}\right)^{1 / 2} \int_{-1}^{1}\left(\frac{1+\xi}{1-\xi}\right)^{1 / 2} \frac{f(\xi) d \xi}{\xi-x} \\
& +\frac{A}{\pi}\left(\frac{1-x}{1+x}\right)^{1 / 2} \frac{1}{(q-x)}
\end{aligned}
$$

or

$$
\begin{aligned}
\gamma(x)= & -\frac{1}{\pi}\left(\frac{1+x}{1-x}\right)^{1 / 2} \int_{-1}^{1}\left(\frac{1-\xi}{1+\xi}\right)^{1 / 2} \frac{f(\xi) d \xi}{\xi-x} \\
& +\frac{A}{\pi}\left(\frac{1+x}{1-x}\right)^{1 / 2} \frac{1}{(q-x)}
\end{aligned}
$$

where $A$ is an arbitrary constant.

3. If $\gamma$ is unbounded at both endpoints but absolutely integrable in a neighborhood of each endpoint, then

$$
\begin{aligned}
\gamma(x)= & -\frac{1}{\left(1-x^{2}\right)^{1 / 2}} \cdot \frac{1}{\pi} \int_{-1}^{1} \frac{\left(1-\xi^{2}\right)^{1 / 2} f(\xi) d \xi}{\xi-x} \\
& +\frac{A}{\pi\left(1-x^{2}\right)^{1 / 2}}+\frac{B}{\pi\left(1-x^{2}\right)^{1 / 2}(q-x)},
\end{aligned}
$$

where $A$ and $B$ are arbitrary constants.

Proof. It is readily verified in each case that the solution satisfies equation (2.1) and the additional conditions imposed at the endpoints. 口 
We note that we may obtain a unique solution for $\gamma$ by imposing one additional condition on the solution in case 2) such as

$$
\int_{-1}^{1} \gamma(x) d x=A_{1}
$$

and, in case 3) a pair of conditions such as

$$
\int_{-1}^{1} \gamma(x) d x=B_{1} \quad \text { and } \quad \int_{-1}^{1} x \gamma(x) d x=B_{2}
$$

Remark 2.2. If, in equation (2.1) instead of $f(x)$ we have $f(x, y)$ where $y$ is looked upon as a parameter in the interval $[-1,1]$, then in equations (2.11) $-(2.13)$ we replace the arbitrary constants $A$ and $B$ by $A(y)$ and $B(y)$ respectively.

3. Bisingular integral equations of the first kind on the square. Let us consider now the problem of flow past a rectangular wing. Bisplinghoff et al. [3, Section 5.5] have shown that the flow of an inviscid, incompressible fluid can be reduced to the solution of the bisingular integral equation of the first kind given by

$$
\frac{1}{\pi^{2}} \int_{-1}^{1} \int_{-1}^{1} \frac{\left((\xi-x)^{2}+(\eta-y)^{2}\right)^{1 / 2}}{(\xi-x)(\eta-y)} \gamma(\xi, \eta) d \xi d \eta=f(x, y)
$$

where $(x, y) \in I_{0}^{2}:=(-1,1) \times(-1,1)$. An analytical solution of this equation is not known so that it has to be solved by approximate methods. For a rectangular wing of large span, Bisplinghoff et al. [3, Section 5.5] have shown that (3.1) can be replaced by

$$
\frac{1}{\pi^{2}} \int_{-1}^{1} \int_{-1}^{1} \frac{\gamma(\xi, \eta) d \xi d \eta}{(\xi-x)(\eta-y)}=f(x, y)
$$

$(x, y) \in I_{0}^{2}$, which is amenable to analytic solution. Indeed, one of us (IKL) has given all the solutions of this equation in the class of absolutely integrable functions on $I_{0}^{2}$ when $f$ is Hölder continuous on $I_{c}^{2}$, see $[\mathbf{1}]$ and $[\mathbf{2}]$. The purpose of this paper is to consider analytic 
solutions of (3.2) in a wider class of functions. This will correspond to the so-called ejection problem when there is only one singularity (source) in one coordinate direction.

To be more specific, suppose $q$, where $-1<q<1$ is a given point on the $0 x$ axis, and let $H_{q}^{*}(x, y)$ denote the class of functions which may be written in the form

$$
\phi(x, y)+\psi(x, y) /(q-x)
$$

where $\phi, \psi$ are Hölder continuous on all compact regions of $I_{0}^{2}$ but are absolutely integrable over $I_{0}^{2}$. We shall now given analytic solutions of (3.2) under varying conditions but in order to avoid the problem of having to pick out a unique solution, we shall impose additional conditions so that a unique solution is always obtained.

Theorem 3.1. Suppose $f$ is Hölder continuous on $I_{c}^{2}$. A solution of equation (3.2) which is bounded along the lines $x= \pm 1, y= \pm 1$ in the class $H_{q}^{*}(x, y)$ on $I_{0}^{2}$ is unique and is given by

$$
\begin{aligned}
\gamma(x, y)= & \frac{\left(1-x^{2}\right)^{1 / 2}\left(1-y^{2}\right)^{1 / 2}}{\pi^{2}} \\
& \cdot \int_{-1}^{1} \int_{-1}^{1} \frac{f(\xi, \eta) d \xi d \eta}{\left(1-\xi^{2}\right)^{1 / 2}\left(1-\eta^{2}\right)^{1 / 2}(\xi-x)(\eta-y)} \\
& -\frac{\left(1-x^{2}\right)^{1 / 2}\left(1-y^{2}\right)^{1 / 2}}{\pi(q-x)} \\
& \cdot \int_{-1}^{1} \frac{d \xi}{\left(1-\xi^{2}\right)^{1 / 2}}\left(\frac{1}{\pi} \int_{-1}^{1} \frac{f(\xi, \eta) d \eta}{\left(1-\eta^{2}\right)^{1 / 2}(\eta-y)}\right),
\end{aligned}
$$

provided that

$$
\int_{-1}^{1} \frac{f(x, \eta) d \eta}{\left(1-\eta^{2}\right)^{1 / 2}}=0, \quad-1 \leq x \leq 1
$$

Proof. We can write (3.2) as

$$
\frac{1}{\pi} \int_{-1}^{1} \frac{d \eta}{\eta-y}\left(\frac{1}{\pi} \int_{-1}^{1} \frac{\gamma(\xi, \eta) d \xi}{\xi-x}\right)=f(x, y)
$$


which can be rewritten as

$$
\frac{1}{\pi} \int_{-1}^{1} \frac{\psi(x, \eta)}{\eta-y} d \eta=f(x, y)
$$

where

$$
\psi(x, \eta):=\frac{1}{\pi} \int_{-1}^{1} \frac{\gamma(\xi, \eta) d \xi}{\xi-x}
$$

Recalling Remark 2.1, we can consider (3.6) as a one-dimensional singular integral equation for $\psi$ with a parameter $x$. From (2.4) and (2.5), we have

$$
\psi(x, y)=-\frac{\left(1-y^{2}\right)^{1 / 2}}{\pi} \int_{-1}^{1} \frac{f(x, \eta) d \eta}{\left(1-\eta^{2}\right)^{1 / 2}(\eta-y)},
$$

provided that

$$
\int_{-1}^{1} \frac{f(x, \eta) d \eta}{\left(1-\eta^{2}\right)^{1 / 2}}=0
$$

which is, of course, (3.4). That $\psi$ is Hölder continuous on $I_{c}^{2}$ follows from arguments similar to those given in Muskhelishvili [7, Section 32]. Returning to (3.7) we consider $\psi$ as known (with "parameter" $\eta$ ) and we can solve it for $\gamma$ in the class of functions $H_{q}^{*}(x, y)$ using Theorem 2.1, case 1 ). From equation (2.10), we obtain

$$
\begin{aligned}
\gamma(x, \eta)= & -\frac{\left(1-x^{2}\right)^{1 / 2}}{\pi} \int_{-1}^{1} \frac{\psi(\xi, \eta) d \xi}{\left(1-\xi^{2}\right)^{1 / 2}(\xi-x)} \\
& +\frac{\left(1-x^{2}\right)^{1 / 2}}{q-x} \cdot \frac{1}{\pi} \int_{-1}^{1} \frac{\psi(\xi, \eta) d \xi}{\left(1-\xi^{2}\right)^{1 / 2}} .
\end{aligned}
$$

Substituting for $\psi$ in this equation and using (3.8), we recover (3.3).

We shall now show that the result of solving (3.2) will be the same if we rewrite it as

$$
\frac{1}{\pi} \int_{-1}^{1} \frac{\phi(\xi, y) d \xi}{\xi-x}=f(x, y)
$$


say, where

$$
\phi(\xi, y):=\frac{1}{\pi} \int_{-1}^{1} \frac{\gamma(\xi, \eta) d \eta}{\eta-y} .
$$

Solving equation (3.11) in the class $H_{q}^{*}(x, y)$, using equation (2.10) of theorem 2.1 gives

$$
\begin{aligned}
\phi(x, y)= & -\frac{\left(1-x^{2}\right)^{1 / 2}}{\pi} \int_{-1}^{1} \frac{f(\xi, y) d \xi}{\left(1-\xi^{2}\right)^{1 / 2}(\xi-x)} \\
& +\frac{\left(1-x^{2}\right)^{1 / 2}}{q-x} \cdot \frac{1}{\pi} \int_{-1}^{1} \frac{f(\xi, y) d \xi}{\left(1-\xi^{2}\right)^{1 / 2}}
\end{aligned}
$$

With $\phi$ given by (3.13), we now solve equation (3.12) for $\gamma$, assuming that $\gamma$ is bounded at both endpoints. From (2.4) we again obtain (3.3), but (2.5) gives this solution subject to the condition that

$$
\begin{aligned}
\int_{-1}^{1} \frac{d \eta}{\left(1-\eta^{2}\right)^{1 / 2}}\{- & \frac{\left(1-x^{2}\right)^{1 / 2}}{\pi} \int_{-1}^{1} \frac{f(\xi, \eta) d \xi}{\left(1-\xi^{2}\right)^{1 / 2}(\xi-x)} \\
& \left.+\frac{\left(1-x^{2}\right)^{1 / 2}}{q-x} \cdot \frac{1}{\pi} \int_{-1}^{1} \frac{f(\xi, \eta) d \xi}{\left(1-\xi^{2}\right)^{1 / 2}}\right\}=0
\end{aligned}
$$

for all $x \in[-1,1]$. Now (3.14) can be written in the form

$$
-F(x)+A /(q-x)=0, \quad \text { for all } x \in(-1,1), \quad x \neq q,
$$

where

$$
F(x):=\frac{1}{\pi} \int_{-1}^{1} \frac{d \xi}{\left(1-\xi^{2}\right)^{1 / 2}(\xi-x)}\left(\int_{-1}^{1} \frac{f(\xi, \eta) d \eta}{\left(1-\eta^{2}\right)^{1 / 2}}\right)
$$

and

$$
A:=\int_{-1}^{1} \int_{-1}^{1} \frac{f(\xi, \eta) d \xi d \eta}{\left(1-\xi^{2}\right)^{1 / 2}\left(1-\eta^{2}\right)^{1 / 2}} .
$$

In order that $(3.15)$ be satisfied, it follows that we must have both $F(x)=0$ on $(-1,1)$ and $A=0$. In order to have $F(x)=0$ on $(-1,1)$, it follows that

$$
\int_{-1}^{1} \frac{f(\xi, \eta) d \eta}{\left(1-\eta^{2}\right)^{1 / 2}}=B
$$


say, for some constant $B$. In that case (3.17) implies that

$$
0=B \int_{-1}^{1} \frac{d \xi}{\left(1-\xi^{2}\right)^{1 / 2}}
$$

so that $B=0$. Thus, we obtain condition (3.4), and the theorem is proved. $\quad \square$

Theorem 3.2. The solution of equation (3.2), together with the condition

$$
\int_{-1}^{1} d \xi \frac{1}{\pi} \int_{-1}^{1} \frac{\gamma(\xi, \eta) d \eta}{\eta-y}=A_{1}(y)
$$

in the class $H_{q}^{*}(x, y)$ on $I_{0}^{2}$, which is bounded along the lines $x=1$, $y=1$ and $y=-1$ and absolutely integrable along $x=-1$ is given by

$$
\begin{aligned}
\gamma(x, y)= & \frac{\left(1-y^{2}\right)^{1 / 2}}{\pi^{2}}\left(\frac{1-x}{1+x}\right)^{1 / 2} \\
& \cdot \int_{-1}^{1} \int_{-1}^{1}\left(\frac{1+\xi}{1-\xi}\right)^{1 / 2} \frac{f(\xi, \eta) d \xi d \eta}{\left(1-\eta^{2}\right)^{1 / 2}(\xi-x)(\eta-y)} \\
& -\frac{1}{\pi}\left(\frac{1-x}{1+x}\right)^{1 / 2} \frac{\left(1-y^{2}\right)^{1 / 2}}{(q-x)} \\
& \cdot \int_{-1}^{1} \frac{\left[A_{1}(\eta)+\int_{-1}^{1}((1+\xi) /(1-\xi))^{1 / 2} f(\xi, \eta) d \xi\right] d \eta}{\left(1-\eta^{2}\right)^{1 / 2}(\eta-y)}
\end{aligned}
$$

provided that the conditions

$$
\int_{-1}^{1}\left(1-\eta^{2}\right)^{-1 / 2} f(x, \eta) d \eta=0
$$

for all $x \in[-1,1]$ and

$$
\int_{-1}^{1}\left(1-\eta^{2}\right)^{-1 / 2} A_{1}(\eta) d \eta=0
$$

are satisfied. 
Proof. Let us rewrite equations (3.2) and (3.20) in the form

$$
\frac{1}{\pi} \int_{-1}^{1} \frac{d \eta}{\eta-y}\left(\frac{1}{\pi} \int_{-1}^{1} \frac{\gamma(\xi, \eta) d \xi}{\xi-x}\right)=f(x, y)
$$

and

$$
\frac{1}{\pi} \int_{-1}^{1} \frac{d \eta}{\eta-y}\left(\int_{-1}^{1} \gamma(\xi, \eta) d \xi\right)=A_{1}(y)
$$

respectively. By considering these equations as equations in $y$ with $x$ as a parameter and recalling that we are looking for a solution bounded along $y=+1$ and $y=-1$, we obtain, upon using equations (2.4) and $(2.5)$,

$$
\frac{1}{\pi} \int_{-1}^{1} \frac{\gamma(\xi, y) d \xi}{\xi-x}=-\frac{\left(1-y^{2}\right)^{1 / 2}}{\pi} \int_{-1}^{1} \frac{f(x, \eta) d \eta}{\left(1-\eta^{2}\right)^{1 / 2}(\eta-y)}
$$

provided that (3.22) is satisfied. Again, since (3.23) is satisfied,

$$
\int_{-1}^{1} \gamma(\xi, y) d \xi=-\frac{\left(1-y^{2}\right)^{1 / 2}}{\pi} \int_{-1}^{1} \frac{A_{1}(\eta) d \eta}{\left(1-\eta^{2}\right)^{1 / 2}(\eta-y)}
$$

Consider equation (3.26). By considering this to be of the form (2.1) with parameter $y$, we have, from (2.11), when solving it in the class $H_{q}^{*}(x, y)$, that

$$
\begin{aligned}
\gamma(x, y)= & \frac{\left(1-y^{2}\right)^{1 / 2}}{\pi^{2}}\left(\frac{1-x}{1+x}\right)^{1 / 2} \\
& \cdot \int_{-1}^{1} \int_{-1}^{1}\left(\frac{1+\xi}{1-\xi}\right)^{1 / 2} \frac{f(\xi, \eta) d \xi d \eta}{\left(1-\eta^{2}\right)^{1 / 2}(\xi-x)(\eta-y)} \\
& +\frac{A(y)}{\pi}\left(\frac{1-x}{1+x}\right)^{1 / 2} \frac{1}{q-x} .
\end{aligned}
$$

But, from this equation, upon using

$$
\frac{1}{\pi} \int_{-1}^{1}\left(\frac{1-\xi}{1+\xi}\right)^{1 / 2} \frac{d \xi}{\xi-x}=-1
$$


for all $x \in(-1,1)$, we find

$$
\begin{aligned}
\int_{-1}^{1} \gamma(x, y) d x= & A(y)+\frac{\left(1-y^{2}\right)^{1 / 2}}{\pi} \\
& \cdot \int_{-1}^{1}\left(\frac{1+\xi}{1-\xi}\right)^{1 / 2}\left(\int_{-1}^{1} \frac{f(\xi, \eta) d \eta}{\left(1-\eta^{2}\right)^{1 / 2}(\eta-y)}\right) d \xi .
\end{aligned}
$$

From (3.27), we see that this gives the unknown function $A(y)$ in terms of the given function $A_{1}$ and we obtain, on using (3.28), the result (3.21).

Again, as in the proof of the preceding theorem, it can be shown that we obtain the same solution if we interchange the order of repeated integration in (3.24) and (3.25). The details are omitted.

We conclude this section with one further result. Other results, depending upon the boundedness or unboundedness of the solution along the boundaries $x= \pm 1, y= \pm 1$, may be derived similarly but we shall omit them.

Theorem 3.3. The solution of equation (3.2), subject to the condition

$$
\frac{1}{\pi} \int_{-1}^{1}\left(\int_{-1}^{1} \frac{\gamma(\xi, \eta) d \xi}{\xi-x}\right) d \eta=C(x)
$$

in the class $H_{q}^{*}(x, y)$ on $I_{0}^{2}$, which is bounded along $x= \pm 1$ and unbounded, but absolutely integrable, along $y= \pm 1$ is given by

$$
\begin{aligned}
\gamma(x, y)= & \frac{\left(1-x^{2}\right)^{1 / 2}}{\left(1-y^{2}\right)^{1 / 2} \pi^{2}} \int_{-1}^{1} \int_{-1}^{1} \frac{\left(1-\eta^{2}\right)^{1 / 2} f(\xi, \eta) d \xi d \eta}{\left(1-\xi^{2}\right)^{1 / 2}(\xi-x)(\eta-y)} \\
& -\frac{\left(1-x^{2}\right)^{1 / 2}}{(q-x)\left(1-y^{2}\right)^{1 / 2}} \cdot \frac{1}{\pi^{2}} \int_{-1}^{1} \int_{-1}^{1} \frac{\left(1-\eta^{2}\right)^{1 / 2} f(\xi, \eta) d \xi d \eta}{\left(1-\xi^{2}\right)^{1 / 2}(\eta-y)} \\
& -\frac{\left(1-x^{2}\right)^{1 / 2}}{\left(1-y^{2}\right)^{1 / 2}} \cdot \frac{1}{\pi^{2}} \int_{-1}^{1} \frac{C(\xi) d \xi}{\left(1-\xi^{2}\right)^{1 / 2}(\xi-x)} \\
& +\frac{\left(1-x^{2}\right)^{1 / 2}}{(q-x)\left(1-y^{2}\right)^{1 / 2}} \frac{1}{\pi^{2}} \int_{-1}^{1} \frac{C(\xi) d \xi}{\left(1-\xi^{2}\right)^{1 / 2}}
\end{aligned}
$$


Proof. Use equations (2.8) and (2.10) as in the proof of the preceding theorem. The details are omitted.

\section{REFERENCES}

1. S.M. Belotserkovsky and I.K. Lifanov, Numerical methods for singular integral equations, Nauka, Moscow, 1985, in Russian.

2. - Method of discrete vortices, CRC Press, Boca Raton, 1992.

3. R.L. Bisplinghoff, H. Ashley and R.L. Halfman, Aeroelasticity, Addison-Wesley, Cambridge, 1955.

4. F.D. Gakhov, Boundary value problems, Pergamon, Oxford, 1966.

5. I.K. Lifanov, Singular solutions of singular integral equations and flow injecting for an arbitrary contour, Soviet J. Numer. Anal. Math. Modeling 4 (1989), 239-252.

6. I.K. Lifanov, A.A. Mikhailov and S.V. Titsky, External flow entrainment and boundary layer separation control for thick aerofoils, Soviet J. Numer. Anal. Math. Modeling 6 (1991), 4-11.

7. N.I. Muskhelishvili, Singular integral equations, P. Noordhoff, Groningen, 1953.

8. S. Okada and D. Elliott, Hölder continuous functions and the finite Hilbert transform, Math. Nachr. 169 (1994), 219-233.

9. H.W. Woolard, Thin-airfoil theory of an ejector-flapped wing section, J. Aircraft 12 (1975), 26-33.

10. E.V. Zakharov, I.I. Lifanov and I.K. Lifanov, Singular solutions of singular integral equations in some problems in electrodynamics, Differential Equations 29 (1993), 1323-1328.

Mathematics Department, University of Tasmania, GPO Box 252-37, Hobart, Tasmania 7001, Australia

Department of Mathematics, Joukowski Air Force Engineering Academy, Leningradski Prospect 40, 125190 Moscow GSP47, Russia

Universidade de Madeira, Colégio dos Jesuitas, largo do Colégio, 9000 Funchal, Madeira, Portugal 